\title{
Reflets
}

Revue ontaroise d'intervention sociale et communautaire

\section{La promotion de l'activité physique : contrer la sédentarité}

\section{Natalie Champion et Nancy Barreira}

Volume 6, numéro 1, printemps 2000

Approches d’intervention : définir et renouveler nos pratiques

URI : https://id.erudit.org/iderudit/026306ar

DOI : https://doi.org/10.7202/026306ar

Aller au sommaire du numéro

Éditeur(s)

Reflets : Revue ontaroise d'intervention sociale et communautaire

ISSN

1203-4576 (imprimé)

1712-8498 (numérique)

Découvrir la revue

Citer cet article

Champion, N. \& Barreira, N. (2000). La promotion de l'activité physique :

contrer la sédentarité. Reflets, 6(1), 220-225. https://doi.org/10.7202/026306ar

Tous droits réservés (C) Reflets : Revue ontaroise d'intervention sociale et communautaire, 2000
Ce document est protégé par la loi sur le droit d'auteur. L'utilisation des services d'Érudit (y compris la reproduction) est assujettie à sa politique d'utilisation que vous pouvez consulter en ligne.

https://apropos.erudit.org/fr/usagers/politique-dutilisation/ 


\title{
La promotion de l'activité physique : contrer la sédentarité
}

\author{
Natalie Champion, \\ B.Sc., B.Sc.inf. (en cours) et
}

Nancy Barreira, $I A A, B$. Sc.inf. (en cours) École des sciences infirmières, Université d' Ottawa ${ }^{1}$

Depuis 1993, le Service de la santé d'Ottawa-Carleton, l'École des sciences infirmières de l'Université d'Ottawa et le Conseil scolaire catholique du district Centre-Est (CSCDCE), se sont engagés dans un projet de partenariat nommé «École en santé». Son but est de créer, au sein des écoles, un milieu physique et social favorable à la santé. Suite à une évaluation des besoins, la promotion de l'activité physique a été choisie comme thème de cette initiative.

Depuis ses débuts, ce partenariat a connu de nombreux succès. Visant d'abord les élèves, les activités se sont multipliées au fil des années pour y inclure la famille. Au cours de l'année scolaire 1998-1999, dans le cadre d'un stage en santé communautaire au Service de la santé d'Ottawa-Carleton, des étudiantes de l'École des sciences infirmières de l'Université d'Ottawa ont complété une évaluation des habitudes d'activité physique des familles dont les enfants fréquentaient une école du CSCDCE. Cet article a pour but d'esquisser les résultats de ce sondage, de discuter du problème de la sédentarité chez les jeunes, d'aborder la question de l'activité physique familiale et, enfin, de présenter les activités entreprises à l'école Ste-Bernadette.

Selon la littérature, les attitudes des Canadiens face à l'activité physique ont évolué négativement et de façon dramatique au cours des récentes années (Jansen 1995). Conséquemment, le niveau d'activité physique diminue rapidement dès un jeune âge 
(Fondation des maladies du cœur en Ontario 1996). Seulement la moitié des jeunes ontariens âgés entre 12 et 15 ans satisfont aux normes pour être reconnus physiquement «actifs», c'est-àdire faire de l'activité physique vigoureuse et assez fréquemment pour activer leur système cardio-vasculaire. Selon le Guide d'activité physique canadien pour une vie active saine (1999), il faut une heure d'activité physique par jour pour demeurer en forme ou améliorer sa santé et ce, pour tous les groupes d'âge.

Les résultats du sondage effectué auprès de parents de l'école Ste-Bernadette, au printemps 1999 (Roy 1999), ont révélé que la majorité des parents (93\%) et des enfants (95\%) aiment faire de l'activité physique. De plus, $84 \%$ des parents en font avec leur enfant.Toutefois, le manque de temps et les coûts sont des facteurs qui, selon les parents, les empêchent de faire des activités avec leurs enfants. Cette étude a aussi révélé que les enfants de ce milieu, âgés de 4 à 8 ans, ont besoin de très peu d'encouragement pour faire de l'activité physique. Bien que nous sachions que le taux d'activité physique diminue à l'adolescence, nos données démontrent qu'à partir de l'âge de 9 ans, les filles ont besoin de plus d'encouragement que les garçons pour faire de l'activité physique.

Nous savons qu'aujourd'hui la technologie (télévision, ordinateur, jeux électroniques, etc.) incitent davantage à la sédentarité. Plus du tiers des parents ayant répondu au sondage étaient d'avis que leur enfant passait trop de temps à se divertir de cette façon. En effet, $36 \%$ des enfants de l'école passaient trois heures ou plus par jour devant la télévision le samedi et le dimanche et $79 \%$, deux heures ou plus. Selon une étude publiée récemment dans la version Internet de Pediatrics (www.pediatrics.org), les enfants regardent trop de télévision, en particulier à l'heure du coucher, ce qui entraîne des troubles de sommeil. Il va sans dire que cela peut engendrer des troubles de rendement scolaire et de comportement. Bien que la télévision semble calmer les adultes, elle a l'effet contraire chez les enfants (Owens et al. 1999).

Les filles se retrouvent encore plus à risque de sédentarité que les garçons, étant donné que les différences de sexe, les niveaux 
de forme physique, les programmes et les opportunités favorisent les garçons dès un jeune âge. Selon Jansen (1995), cette réalité est reliée au statut économique, à l'ethnie, à la disponibilité de programmes offerts et au manque de modèles de rôles féminins. Ces facteurs affectent directement la motivation des filles face à l'activité physique et leur degré d'habileté. Par contre, les filles qui reçoivent du soutien et de l'encouragement pour faire de l'activité physique, qui ont des modèles positifs et qui fréquentent des écoles leur fournissant des opportunités d'activité physique égales aux garçons, récoltent autant de succès que ces derniers (Jansen 1995).

Selon Nader (1996), la famille est une source majeure d'influence et de modelage pour les comportements de santé des enfants. L'influence et le niveau de connaissances des parents ont des conséquences importantes sur l'attitude des enfants face à leur santé (Petchers 1997). Les habitudes familiales sont en effet un facteur déterminant pour l'activité physique chez les enfants (DiLorenzo 1998; Petchers et al. 1987). De plus, lorsque le parent participe à des activités physiques avec son enfant, ce dernier acquiert une perception de ses compétences et une attitude positive envers l'activité physique (Brustad 1996). En d'autres mots, si les parents sont actifs, l'enfant a plus de chance de l'être à son tour (Brustad 1996). Saunders et al. (1997) affirment que les facteurs déterminants de l'activité physique chez les jeunes sont associés aux facteurs héréditaires, au soutien des pairs, à l'activité parentale et à son soutien, à la forme physique personnelle, au temps passé dehors et à la perception de son efficacité.

Il existe une multitude de bienfaits à l'activité physique, tels que la performance améliorée à l'école à travers une meilleure concentration, une augmentation du niveau d'attention, de l'estime de soi et de l'efficacité, et une amélioration de la forme physique et de la tenue corporelle (Fondation des maladies du cœur en Ontario 1996). Les jeunes plus actifs se retrouvent en meilleure santé, démontrent moins d'agressivité, sont moins vulnérables au stress, sont plus indépendants et développent de meilleures habitudes sociales (Jansen 1995). 
Dans le cadre de notre projet, nous retrouvons ces bienfaits chez l'enfant, mais bien entendu, l'enfant ne peut être visé sans considérer sa famille. Par conséquent, les activités entreprises par les étudiantes de l'École des sciences infirmières étaient concentrées sur la mise en œuvre d'un programme de promotion de l'activité physique au sein de la famille. Suite à une recension des écrits, nous avons décidé d'adapter une version du programme intitulé "Famille en mouvement», du Bureau de santé publique Middlesex-London. Ce programme avait comme but de changer ou d'améliorer les habitudes familiales face à l'activité physique, de diminuer le taux de sédentarité, de donner aux membres de la famille le goût de faire de l'activité physique afin qu'ils continuent à en faire en dehors des activités scolaires et enfin, d'encourager et de promouvoir les activités familiales.

Le programme «Famille en mouvement» s'est déroulé à l'école Ste-Bernadette du 15 novembre au 6 décembre 1999, soit une période de trois semaines. Les élèves étaient conviés à faire quinze minutes d'activité physique, trois fois par semaine avec un des membres de leur famille. L'activité choisie devait accélérer le rythme cardiaque et durer au moins quinze minutes. Par la suite, les élèves notaient toutes les activités physiques qu'ils avaient pratiquées sur un billet intitulé "Nous l'avons fait», qui leur était remis au début de chaque semaine du programme. Les billets de chacune des classes de l'école se distinguaient grâce à l'image d'un athlète particulier qui y était imprimée. Par exemple, la maternelle était représentée par une patineuse artistique, la troisième année par un joueur de hockey, etc. Lorsque trois activités étaient notées sur le billet, l'enfant le remettait à son enseignante, accumulant ainsi un point pour sa classe. À la fin de chaque semaine, un membre du comité "Famille en mouvement» additionnait les points accumulés par chacune des classes et les inscrivait sur un babillard à l'école intitulé «Vers le flambeau olympique : Sydney 2000", où nous retrouvions les images d'athlètes représentant chacune des classes. Le but ultime était d'accumuler assez de points pour pouvoir allumer le flambeau olympique à la fin des trois semaines du programme «Famille en mouvement». 
Un bulletin d'information fut envoyé aux enseignantes et aux enseignants de l'école, décrivant les objectifs du programme, son fondement, ses bienfaits, le rôle de l'école et le mode de participation. De plus, un dépliant envoyé aux parents de l'école décrivait l'importance de leur rôle dans le développement des habitudes de vie saines, les raisons pour participer, la durée du programme et ses bienfaits. Plusieurs associations de sports amateurs furent sollicitées par les étudiantes, afin d'obtenir du matériel promotionnel et des récompenses pour susciter la participation des élèves. À partir du matériel obtenu, des trousses pour chaque salle de classe ont été distribuées aux enseignantes. Ces trousses contenaient des informations sur les consignes du programme, les billets «Nous l'avons fait» et les récompenses pour les trois semaines du programme. Une série d'annonces promotionnelles sous forme de comptines, a été développée et transmise à chaque matin à l'interphone (intercom) de l'école.

L'analyse des résultats de "Famille en mouvement» a révélé que les élèves de la maternelle et les membres de leur famille ont participé le plus activement au programme tandis que les jeunes des $7^{\mathrm{e}}$ et $8^{\mathrm{e}}$ années ont participé le moins. Ce résultat ne surprend pas puisqu'on pouvait l'anticiper à partir de la recension des écrits sur le sujet. À Ste-Bernadette, «Famille en mouvement» a été une stratégie gagnante auprès des plus jeunes. Toutefois, cela nous signale qu'il faudra développer des initiatives et des projets mieux ciblés afin de motiver les élèves plus âgés.

En plus de «Famille en mouvement», les étudiantes de l'École des sciences infirmières ont fait des activités au sein de l'école, telles que des ateliers sur la nutrition et le tabagisme. De plus, un deuxième dépliant fut élaboré à l'intention des parents, ciblant cette fois-ci la promotion d'activités physiques chez les jeunes filles, étant donné leurs risques plus élevés de sédentarité.

Le bilan de cette recherche-action reste à faire puisque certaines des interventions entreprises par les étudiantes de l'École des sciences infirmières et le comité "École en santé» à l'école SteBernadette pour réduire la sédentarité chez les jeunes, promouvoir l'activité physique familiale et inciter les filles à l'activité physique se continuent. Tout comme l'indique l'étude de Petchers et al. 
(1987), nous avons toutefois pu constater que des efforts combinés entre les parents et l'école sont l'approche idéale pour une santé optimale des enfants!

\section{Bibliographie}

BRUSTAD, R. (1996). «Attraction to Physical Activity in Urban School Children :Parental Socialization and Gender Influence", Research Quarterly for Exercise and Sport, vol. 67, no 3, 316-323.

DILORENZO et al. (1998). «Determinants of Exercise Among Children. II.A Longitudinal Analysis», Preventive Medicine, vol. 27, 470-477.

SANTÉ CANADA. (1999) Guide d'activité physique canadien pour une vie active saine, Ottawa, Santé Canada.

HEART AND STROKE FOUNDATION OF ONTARIO (1996). Position on Reforms Concerning Physical and Health Education at the Secondary School Level, Ontario, Heart and Stroke Foundation.

JANSEN, M. (1995). "Women and Girls in Sport and Physical Activity», Canadian Women Studies, vol. 15, no 4, 1-4.

NADER, P. et al. (1996). «The Effect of Adult Participation in a School-Based Family Intervention to Improve Children's Diet and Physical Activity : the Child and Adolescent Trial for Cardiovascular Health», Preventive Medicine, vol. 25, 455-464.

OWENS,J., R. MAXIM, M.MCGUINN, C.NOBILE, M. MSALL et A.ALARIO (1999). «Televisionviewing Habits and Sleep Disturbance in School Children», Pediatrics, vol. 104, no 3, 27.

PETCHERS, M., E. HIRSCH et B. BLOCH (1987). «The Impact of Parent Participation on the Effectiveness of a Heart Health Curriculum», Health Education Quarterly, vol. 14, no 4, 449-460.

ROY, J. (1999). L'activité physique des familles de l'école Ste-Bernadette. Résultats d'un sondage auprès des parents au printemps 1999, Ottawa, Service de la santé de la Région d'Ottawa-Carleton.

SAUNDERS, R.P., R.P.PATE, G. FELTON, M.DOWDA, M.C.WEINRICH etT.BARANOWSKI (1997). «Development of Questionnaires to Measure Psychosocial Influences on Children's Physical Activity", Preventive Medicine, no 26, 241-247.

1. Nous aimerions remercier l'école Ste-Bernadette, son directeur, M. Richard Laplante, son personnel enseignant et toutes les familles de l'école. Nous tenons également à remercier notre professeure clinique, Denise Hébert, de son accompagnement au cours de ce stage clinique. 